\title{
Association of xeroderma pigmentosum group D (Asp312Asn, Lys751Gln) and cytidine deaminase (Lys27GIn, Ala70Thr) polymorphisms with outcome in Chinese non-small cell lung cancer patients treated with cisplatin-gemcitabine
}

\author{
M. Zhou*, Y.J. Ding*, Y. Feng, Q.R. Zhang, Y. Xiang and H.Y. Wan \\ Department of Respiratory Medicine, Ruijin Hospital, \\ Shanghai Jiao Tong University School of Medicine, Shanghai, China \\ *These authors contributed equally to this study. \\ Correspondence author: H.Y. Wan \\ E-mail: cnshzhoumin@live.cn
}

Genet. Mol. Res. 13 (2): 3310-3318 (2014)

Received January 29, 2013

Accepted January 16, 2014

Published April 29, 2014

DOI http://dx.doi.org/10.4238/2014.April.29.9

ABSTRACT. Xeroderma pigmentosum group D (XPD) plays a key
role in the repair of DNA and platinum resistance lesions. Cytidine
deaminase (CDA) genes determine the velocity of gemcitabine
catalysis. This study aimed to investigate the relationship between XPD
and CDA genotypes and outcome in non-small lung cancer (NSCLC)
patients. We used polymerase chain reaction-restriction fragment length
polymorphism to evaluate genetic polymorphisms of XPD (Asp312Asn
and Lys751Gln) and CDA (Lys27Gln and Ala70Thr) in 93 NSCLC
patients treated with a cisplatin-gemcitabine regimen. There were no
significant correlations between the XPD polymorphisms Asp312Asn
and Lys751Gln with clinical benefits (P > 0.05). Time to progression
(TTP) did not differ between patients with wild type genotypes and
those heterozygous for the single nucleotide polymorphism loci of 
XPD. However, a significant difference was observed in overall survival (OS) between XPD Asp312Asp and XPD Asp312Asn individuals (20.0 vs 12.4 months, $\mathrm{P}=0.04$ ). Furthermore, the $\mathrm{OS}$ of patients with wild type genotypes was longer (20.5 months) than that of patients carrying the XPD 751Lys/Gln polymorphism (11.5 months). No significant differences in TTP or OS were observed in patients carrying different genotypes of CDA Lys27Gln, and no mutations were observed at the CDA Ala70Thr site. These results provide suggestive evidence of a favorable effect for the XPD 312Asp/Asp and XPD 751Lys/Lys genotypes with respect to overall survival rates in platinum-treated NSCLC patients. However, the CDA 27 polymorphism does not appear to affect the efficacy of gemcitabine.

Key words: Genetic polymorphisms; Cytidine deaminase; Lung cancer; Xeroderma pigmentosum complementary group D

\section{INTRODUCTION}

Lung cancer is a leading cause of cancer-related deaths in China. Non-small cell lung cancer (NSCLC) accounts for more than $80 \%$ of primary lung cancers, and because $70-80 \%$ of NSCLC patients are diagnosed at an advanced stage, most patients are treated with cytotoxic chemotherapy. Currently, most lung cancer patients are treated with platinum-based chemotherapy. Although chemotherapy based on platinum regimens is the main treatment for the disease, the five-year survival rate from either small cell lung cancer (SCLC) or NSCLC remains no better than $15 \%$ (Jemal et al., 2002). Although the major prognostic determinant for lung cancer is the stage at presentation, there are nonetheless differences in survival for patients presenting with the same stage of the disease. More accurate prognosis assessments should be helpful in deciding therapeutic options, opening up the possibility for patient-tailored decisions with respect to drug selection.

With respect to NSCLC, new cytotoxic drugs, such as gemcitabine, paclitaxel, docetaxel, or vinorelbine, used in combination with a platinum compound have shown an absolute 15 to $20 \%$ improvement in survival, favoring chemotherapy over best supportive care. Indeed a four-arm randomized phase III trial showed no obvious differences in response rate, time to progression (TTP), and overall survival (OS) among gemcitabine-cisplatin, paclitaxelcisplatin, docetaxel-cisplatin, and paclitaxel-carboplatin combinations. However, the one-year survival rate was less than $40 \%$ in lung cancer patients who received any chemotherapy, and all of the last-generation doublets appeared to have reached the therapeutic plateau (Schiller et al., 2002). Therefore, appropriate drugs need to be determined for each patient individually. Recently, several pharmacological studies have emerged to identify candidate genes that may account for the cancer- and patient-related components to explain outcome variability.

Among the last-generation doublets, gemcitabine-cisplatin is a cytotoxic drug that is commonly used as first-line therapy in advanced NSCLC. There is some evidence that transcriptional activity and polymorphisms of genes encoding proteins involved in the metabolic action of gemcitabine could be used to determine which patients can most benefit from gemcitabine-based chemotherapy (Fukunaga et al., 2004). Cytidine deaminase (CDA) is the key enzyme that determines gemcitabine clearance, since it catalyzes its degradation (Danesi et 
al., 2009). Because platinum compounds produce adducts and break the double helix of DNA, individual variability of DNA repair correlates with the efficacy of cytotoxic agents. Xeroderma pigmentosum D (XPD) is an ATP-dependent DNA helicase that is involved in DNA repair of the nucleotide excision repair (NER) pathway (Lunn et al., 2000). Several studies have identified XPD polymorphisms, and some studies have indicated that variation in the XPD gene may influence clinical benefits of platinum-based therapy (Wlodarczyk and Nowicka, 2012).

Based on these investigations, we assessed the association of XPD and CDA polymorphisms with clinical outcome in NSCLC in a Chinese population.

\section{MATERIAL AND METHODS}

\section{Patient recruitment and follow-up}

This study included 93 patients with advanced NSCLC (stage IIIB-IV), who were diagnosed from January 2006 to August 2010. All patients were referred for chemotherapy at the Respiratory Department of Ruijin Hospital, affiliated to the Shanghai Jiao Tong University School of Medicine. Every patient's diagnosis was confirmed by histopathology or cytology. The recommendations of the Declaration of Helsinki for biomedical research involving human subjects were followed.

Clinical data were systematically recorded upon admission, including age, gender, smoking status, tumor histology, and clinical stage. We also recorded the treatment regimen, objective response, relapses, TTP, adverse effects produced by the therapy, and death. The assessment method used to determine baseline disease status was consistently used for efficient evaluation throughout the patient's follow-up. All patients signed written informed consent before study entry.

\section{Chemotherapy regimens}

The 93 NSCLC patients received $1250 \mathrm{mg} / \mathrm{m}^{2}$ gemcitabine by $i v$ mode over $30 \mathrm{~min}$ on days one and eight in combination with $75 \mathrm{mg} / \mathrm{m}^{2}$ cisplatin infused over $60 \mathrm{~min}$ given on day one every three weeks. All patients were treated for two to six cycles.

\section{DNA extraction and genotyping}

Three-milliliter blood samples were collected for genotyping at the time of study entry before drug administration. Genomic DNA was extracted from blood using a DNA extraction kit. Genotype distributions were determined with polymerase chain reaction-restriction fragment length polymorphism (PCR-RFLP), which included XPD Asp312Asn, XPD Lys751Gln, CDA Lys27Gln, and CDA Ala70Thr. Ten-microliter PCR systems contained $0.3 \mu \mathrm{L}$ template DNA, $1 \mu \mathrm{L}$ 10X Taq buffer, $0.5 \mu \mathrm{L}$ dNTP, $0.5 \mathrm{pM}$ primer, and $0.3 \mathrm{U}$ Taq polymerase. The PCR (Canada BBI company) amplification conditions were as follows: denaturation for 5 min, and then $95^{\circ} \mathrm{C}$ for $30 \mathrm{~s}$, annealing for $45 \mathrm{~s}, 72^{\circ} \mathrm{C}$ for $60 \mathrm{~s}$ ( 40 cycles), followed by $72^{\circ} \mathrm{C}$ for $6 \mathrm{~min}$. The annealing temperature for the XPD Asp312Asn and CDA Ala70Thr primers was $60^{\circ} \mathrm{C}$, and $58^{\circ} \mathrm{C}$ for the XPD Lys751Gln and CDA Asp312Asn primers. Single nucleotide polymorphisms (SNPs) were characterized through PCR-RFLP by digesting the amplified XPD Asp312Asn with MboII, the XPD Lys751Gln with Eam1104, the CDA Lys27Gln with MboII, and the CDA Ala70Thr with CpoI. The MboII-restricted product of XPD had an 
expected size of $114 \mathrm{bp}+29 \mathrm{bp}$ for the 312Asn allele and $143 \mathrm{bp}$ for the 312Asp allele. The Eam 1104I-restricted product of XPD had an expected size of $368 \mathrm{bp}$ for the Lys751 allele and $130 \mathrm{bp}+238 \mathrm{bp}$ for the Gln751 allele. The MboII-restricted product of CDA had an expected size of $129 \mathrm{bp}$ for Lys 27 and $48 \mathrm{bp}+81 \mathrm{bp}$ for Gln27. The CpoI-restricted product of CDA had an expected size of $300 \mathrm{bp}$ for Ala70 and $181 \mathrm{bp}+119 \mathrm{bp}$ for Thr70. The PCR products were evaluated by restriction enzyme digestion overnight at $37^{\circ} \mathrm{C}$, and were then run on $2 \%$ agarose gel. Mutations of the genes were determined by sequencing.

\section{Statistical analysis}

Clinical information and gene frequencies were compared using the chi-squared test. The XPD and CDA polymorphisms were analyzed according to haplotype. The objective of the analysis was to determine the association between the polymorphisms and demographics, pretreatment characteristics, and outcome data. Pearson chi-squared tests were used to determine the relationship between each categorical variable and XPD genotype. The KaplanMeier method was used to plot TTP and OS, and the log-rank test was used to compare curves in univariate analyses. Factors included in univariate analyses were genotypes, gender (male versus female), performance status, age, clinical stage (IIIB versus IV), histology (adenocarcinoma, including bronchioloalveolar versus squamous, and large cell carcinoma), and smoking history (never versus former and current smokers). Data were analyzed using the SPSS13.0 (IBM) software. Statistical significance was set at $\mathrm{P}<0.05$.

\section{RESULTS}

\section{Patient characteristics and treatment}

A total of 93 Chinese patients were enrolled in the study. The majority of patients were in the stage IIIB phase $(59.60 \%)$, whereas 40 patients $(40.40 \%)$ were in the stage IV phase (Table 1).

\begin{tabular}{lc} 
Table 1. Clinical characteristics of the patients. \\
\hline \\
\hline No. patients & No. of patients (\%) \\
Median age, years (range) & 93 \\
Gender & $61.5 \pm 1.2$ \\
Male & $56(60.22)$ \\
Female & $37(39.78)$ \\
Smoking status & \\
Smoking & $49(52.69)$ \\
Never smoking & $44(47.31)$ \\
Clinical stage & \\
IIIB & $53(56.99)$ \\
IV & $40(43.01)$ \\
ECOG PS & \\
1 & $43(46.24)$ \\
2 & $50(53.76)$ \\
Histology & \\
Adeno & $70(75.27)$ \\
Squamous & $21(22.58)$ \\
Large cell & $2(2.15)$ \\
\hline
\end{tabular}




\section{Genotype information}

The distribution of all polymorphisms and the allelic frequencies are shown in Table 2. The frequency of the XPD 312Asp/Asp genotype was 80.65\%, whereas the frequency of Asp/ Asn heterozygotes was $19.35 \%$. With respect to the XPD 751 polymorphism, the wild type was found in $81.72 \%$ of cases, and the heterozygous Lys/Gln variant had a frequency of $18.28 \%$. With respect to the CDA27 polymorphisms, the frequencies of the wild type (Lys/Lys) and Lys/ Gln genotypes were $79.57 \%$ and $20.43 \%$, respectively, and all the patients with wild type CDA genotypes showed 70Ala/Ala. After approximately two to four cycles of chemotherapy, we evaluated the correlation between XPD and CDA genotypes and objective response.

\begin{tabular}{lcc}
\multicolumn{2}{l}{ Table 2. Xeroderma pigmentosum (XPD) and cytidine deaminase (CDA) Polymorphisms. } \\
\hline Genotype & No. of patients & Allelic frequencies \\
\hline XPD Asp312Asn & $75(80.65 \%)$ & \\
G/G & $18(19.35 \%)$ & G (Asp) 0.91 \\
A/G & $76(81.72 \%)$ & A (Asn) 0.09 \\
XPD Lys751Gln & $17(18.28 \%)$ & T (Lys) 0.91 \\
T/T & $74(79.57 \%)$ & G (Gln) 0.09 \\
T/G & $19(20.43 \%)$ & A (Lys) 0.89 \\
CDA Lys27Gln & $93(100 \%)$ & C (Gln) 0.11 \\
A/A & 0 & G (Ala) 1.0 \\
A/C & & A (Thr) 0 \\
CDA Ala70Thr &
\end{tabular}

\section{XPD Asp312Asn}

Forty-four percent of the patients with the XPD 312Asp/Asp genotype showed progression of the disease, whereas 55.56\% of patients with the heterozygous Asp/Asn genotype showed disease progression. There was no significant correlation between XPD Asp312Asn and clinical benefit $(\mathrm{P}=0.502)$ (Table 3$)$.

\begin{tabular}{|c|c|c|c|c|}
\hline Genotype & PR No. patients (\%) & SD No. patients (\%) & PD No. patients (\%) & $\mathrm{P}$ \\
\hline \multicolumn{5}{|c|}{ XPDAsp ${ }^{312}$ Asn } \\
\hline Asp/Asp & $27(36.00 \%)$ & $15(20.00 \%)$ & $33(44.00 \%)$ & \multirow[t]{3}{*}{0.502} \\
\hline Asp/Asn & $4(22.22 \%)$ & $4(22.22 \%)$ & $10(55.56 \%)$ & \\
\hline \multicolumn{4}{|c|}{ XPD Lys $^{751} \mathrm{Gln}$} & \\
\hline Lys/Lys & $26(34.21 \%)$ & $15(19.74 \%)$ & $35(46.05 \%)$ & \multirow[t]{2}{*}{0.517} \\
\hline Lys/Gln & $5(29.41 \%)$ & $4(23.53 \%)$ & $8(47.06 \%)$ & \\
\hline \multicolumn{5}{|c|}{$\mathrm{CDA} \mathrm{Lys}^{27} \mathrm{Gln}$} \\
\hline Lys/Lys & $21(28.38 \%)$ & $17(22.97 \%)$ & $36(48.65 \%)$ & \multirow[t]{2}{*}{0.493} \\
\hline Lys Gln & $8(42.11 \%)$ & $3(15.79 \%)$ & $8(42.11 \%)$ & \\
\hline
\end{tabular}

\section{XPD Lys751GIn}

With respect to the XPD 751 polymorphism, $81.72 \%$ of patients carrying the XPD $751 \mathrm{Lys} / \mathrm{Lys}$ genotype and $53.95 \%$ of wild type patients showed clinical benefits (PR or SD), and $52.94 \%$ of XPD $751 \mathrm{Lys} / \mathrm{Gln}$ patients responded to therapy. There was no significant dif- 
ference among genotypes $(\mathrm{P}=0.517)$ (Table 3$)$.

\section{CDA Lys27GIn}

We evaluated the CDA Lys27Gln genotype in the 93 NSCLC patients. Partial remission was observed in $28.38 \%$ of wild-type patients, whereas $42.11 \%$ of heterozygous patients obtained partial remission. Although the response rate of heterozygous patients was higher than that of wild type patients, the difference was not significant $(\mathrm{P}=0.493)$. Overall, no significant correlation was observed between XPD and CDA genotypes with objective response (Table 3).

\section{Variation in TTP and OS with respect to XPD and CDA genotypes}

The Kaplan-Meier method was used to plot TTP and OS, and the log-rank test was used to compare curves in univariate analyses. No significant differences in TTP were observed with respect to XPD 312, XPD 751, and CDA 27 genotypes.

Table 4 shows correlations between polymorphisms and survival. The median TTP was 11.2 months $(95 \%$ confidence interval $=8.6-13.9$ months $)$ in patients carrying the XPD $312 \mathrm{Asp} /$ Asp genotype, whereas it was 6.8 months in those carrying the Asp/Asn genotype (P $=0.25)$. There was no difference in TTP between wild type genotype patients (10.7 months) and XPD 751Lys/Gln genotype patients (7.0 months). However, the difference in OS between XPD 312Asp/Asp and Asp/Asn patients was significant (20.0 vs 12.4 months, respectively, $\mathrm{P}=$ $0.04)$. In addition, the OS of wild type genotype patients (20.5 months) was longer than that of XPD 751Lys/Gln patients (11.5 months). The univariate analysis using the log-rank test also revealed that smoking status was significantly associated with OS $(\mathrm{P}=0.029)$, whereas age ( $\mathrm{P}$ $=0.62)$, gender $(\mathrm{P}=0.13)$, and histology $(\mathrm{P}=0.54)$ were not prognostic factors. No significant differences in TTP or OS were observed among different genotypes of CDA Lys27Gln.

\begin{tabular}{|c|c|c|c|c|}
\hline Genotype & Median TTP, mo $(95 \% \mathrm{CI})$ & $\mathrm{P}$ & Median OS, mo $(95 \% \mathrm{CI})$ & $\mathrm{P}$ \\
\hline \multicolumn{5}{|c|}{ XPDAsp312Asn } \\
\hline $\mathrm{G} / \mathrm{G}$ & $11.2(8.6-13.9)$ & 0.25 & $20.0(16.3-23.6)$ & 0.04 \\
\hline $\mathrm{A} / \mathrm{G}$ & $6.8(6.1-10.5)$ & & $12.4(11.0-13.9)$ & \\
\hline \multicolumn{5}{|c|}{ XPDLys751Gln } \\
\hline $\mathrm{T} / \mathrm{T}$ & $10.7(8.1-13.5)$ & 0.39 & $20.5(16.9-24.1)$ & 0.03 \\
\hline $\mathrm{T} / \mathrm{G}$ & $7.0(4.6-9.6)$ & & $11.5(9.01-15.2)$ & \\
\hline \multicolumn{5}{|c|}{ CDA Lys27Gln } \\
\hline $\mathrm{A} / \mathrm{A}$ & $10.1(7.2-12.8)$ & 0.62 & $13.4(8.5-18.5)$ & 0.41 \\
\hline $\mathrm{A} / \mathrm{C}$ & $7.8(5.1-10.5)$ & & $11.4(5.7-17.1)$ & \\
\hline
\end{tabular}

\section{DISCUSSION}

Standard treatment for NSCLC involves chemotherapy with a platinum agent and another cytotoxic agent. However, platinum-based therapies often induce drug resistance. Platinum compounds form both intrastrand and interstrand DNA adducts that result in bulky distortion of DNA and destabilization of the double helix, unless these adducts are repaired before the DNA replicates. Although resistance to chemotherapy is multifactorial, DNA repair plays a key role in 
platinum resistance (Booton et al., 2006). DNA repair has been termed a double-edged sword. In mammalian cells, nucleotide excision repair is the major pathway for removing damaged bases from DNA. XPD is an ATP-dependent DNA helicase. It is a multifunctional gene that encodes a component of the transcription factor II H (TF IIH), and participates in p53-mediated apoptotic responses. XPD has dual functions in the cell: nucleotide excision repair and cell cycle regulation through actions on the Cdk-activating kinase (Takenaka et al., 2010). XPD polymorphisms have been known to correlate with DNA repair capacity in various tissues. SNPs in XPD alter its efficiency, which affects the risk of cancer and the outcome of platinum-chemotherapy. There are six SNPs in the XPD gene, located at codons $199,201,312,711$, and 751 . The polymorphisms in codons 199, 201, 312, and 751 may result in amino acid changes. The mutation frequency of the 199 and 201 alleles is only 4\%, while mutations of codons 312 and 751 are more common, prompting our focus on Lys751Gln and Asp312Asn. Codon 312 in XPD is highly conserved, indicating that it may play an important role in the function of the XPD protein. Homozygous cells of an XPD 312Asn mutated protein showed higher DNA repair capacity (Seker et al., 2001). Although codon 751 on the N-terminus of XPD is poorly conserved, it is also associated with differential DNA repair efficiency. In a study of lung cancer patients, the XPD Asp312Asn and Lys751Gln polymorphisms were consistently associated with lower DNA repair capacity as assessed by a cell reactivation assay (Spitz et al., 2001).

In a previous study, we found no significant difference in the mutations XPD Asp312Asn and XPD Lys $751 \mathrm{Gln}$ between lung cancer patients and control groups in a Chinese population. However, cancer risk might be greater in people that carry the Lys751Gln mutation and have a history of smoking. Individuals with both codon 312 and 751 mutations are more likely to develop lung cancer, indicating an interaction between these two sites. In order to investigate whether the SNP of XPD is a valuable predictive factor for platinum-based chemotherapy, we evaluated the role of XPD gene polymorphisms in the survival of advanced-stage NSCLC patients treated with platinum agents. We found that the SNPs XPD Asp312Asn and XPD LyS751Gln did not affect the TTP of the patients. However, patients with wild type genotypes at both sites survived longer than heterozygous patients did.

Gurubhagavatula et al. (2004) also showed that increasing numbers of variant XPD alleles were associated with shorter overall survival. A significant association was also observed between the wild-type genotype for XPD 751 and longer OS in metastatic colorectal cancer patients treated with 5-fluorouracil and oxaliplatin (Park et al., 2001). However, in a metaanalysis, Wei et al. (2011) suggested that XPD Asp312Asn and XPD Lys751Gln were not predictive markers for platinum-based chemotherapy in patients with advanced NSCLC. Gao et al. (2006) suggested that individuals who smoked and had the XPD 312Asp/Asp genotype could be at greater risk of p53 mutations, especially if combined with other polymorphisms that might result in deficient DNA repair. Therefore, perhaps other factors (e.g., smoking, various cancers) also affect DNA repair capacity.

The metabolic inactivation of gemcitabine is catalyzed by CDA. CDA contains two sense mutations: CDA Lys27Gln and CDA Ala70Thr. Our results showed that the frequency of the CDA Lys $27 \mathrm{Gln}$ genotype was $11 \%$ in the Chinese population, which is similar to that observed in African Americans. The mutation rate of CDA Ala70Thr is very low within the Chinese population, and there was no mutation on the CDA Ala70Thr site observed in the lung cancer group. Our results suggested that these CDA mutations did not increase the risk of lung cancer. Therefore, we investigated the relationship between the CDA Lys27Gln poly- 
morphism and the efficacy of gemcitabine. There was no significant difference in TTP or OS among different genotypes of CDA Lys27Gln.

Previous studies investigating CDA SNPs and their predictive role in clinical outcomes have yielded controversial results. Gilbert et al. (2006) observed a significant decrease in the deamination activity of gemcitabine in a CDA variant characterized by the non-synonymous polymorphism of lysine 27 glutamine (Lys27Gln), resulting from an A/ substitution in exon 79. Cells transfected with CDA, and hence showing very high CDA activity, were relatively less sensitive to gemcitabine. Tibaldi et al. (2008) suggested that the CDA 27Lys/Lys genotype predicted a better clinical benefit, a higher response rate, and longer TTP and OS compared to the other CDA genotypes. Another clinical study showed a correlation between mRNA expression of CDA in peripheral blood mononuclear cells and clinical outcome in gemcitabine-treated patients (Bengala et al., 2005). Maring et al. (2010) investigated the impact of the common non-synonymous SNP in the CDA gene, CDA 27, on gemcitabine pharmacokinetics in NSCLC patients. They studied the consequences of this polymorphism on gemcitabine area under the curve (AUC), clearance, and serum half-life. They found that the CDA 27 polymorphism did not have any major consistent or significant impact on gemcitabine pharmacokinetics.

Taken together, our observations suggest that the two SNPs, XPD Asp312Asn and Lys $751 \mathrm{Gln}$, may affect cisplatin resistance and may influence clinical outcomes, especially OS. In contrast, the CDA 27 polymorphism does not appear to affect the efficacy of gemcitabine. Perhaps the different results stem from the limited sample size and race evaluated in the present study. One explanation for our findings may be related to the relatively more complex regulation of the function of the XPD gene. For example, smoking and p53 gene mutations affect the DNA repair capacity of XPD, whereas gemcitabine metabolism is affected by more factors: CDA gene transcription, CDA enzyme activity, and other enzymes involved in gemcitabine metabolism. In addition, it remains controversial whether the SNP of biomarker genotype from blood samples is the same as that obtained from cancer tissues directly. SNPs are inherited from genetic variants harbored by all cells of the body, and although a genotype represents a static value unable to change in response to different environmental contexts, such as exposure to chemotherapy, it may not reflect changes in tumor DNA, such as the loss of heterozygosity. Therefore, more research is needed to establish effective and reliable predictive makers for chemotherapy in patients with advanced NSCLC.

\section{ACKNOWLEDGMENTS}

We would like to thank all the patients who participated in the study. This study was supported by the Shanghai Cancer Foundation of China and from Wu Jie Ping Cancer Foundation.

\section{Conflicts of interest}

The authors declare no conflict of interest.

\section{REFERENCES}

Bengala C, Guarneri V, Giovannetti E, Lencioni M, et al. (2005). Prolonged fixed dose rate infusion of gemcitabine with 
autologous haemopoietic support in advanced pancreatic adenocarcinoma. Br. J. Cancer 93: 35-40.

Booton R, Ward T, Heighway J, Taylor P, et al. (2006). Xeroderma pigmentosum group D haplotype predicts for response, survival, and toxicity after platinum-based chemotherapy in advanced nonsmall cell lung cancer. Cancer 106: 24212427.

Danesi R, Altavilla G, Giovannetti E and Rosell R (2009). Pharmacogenomics of gemcitabine in non-small-cell lung cancer and other solid tumors. Pharmacogenomics 10: 69-80.

Fukunaga AK, Marsh S, Murry DJ, Hurley TD, et al. (2004). Identification and analysis of single-nucleotide polymorphisms in the gemcitabine pharmacologic pathway. Pharmacogenomics J. 4: 307-314.

Gao WM, Romkes M, Siegfried JM, Luketich JD, et al. (2006). Polymorphisms in DNA repair genes XPD and XRCC1 and p53 mutations in lung carcinomas of never-smokers. Mol. Carcinog. 45: 828-832.

Gilbert JA, Salavaggione OE, Ji Y, Pelleymounter LL, et al. (2006). Gemcitabine pharmacogenomics: cytidine deaminase and deoxycytidylate deaminase gene resequencing and functional genomics. Clin. Cancer Res. 12: 1794-1803.

Gurubhagavatula S, Liu G, Park S, Zhou W, et al. (2004). XPD and XRCC1 genetic polymorphisms are prognostic factors in advanced non-small-cell lung cancer patients treated with platinum chemotherapy. J. Clin. Oncol. 22: 2594-2601.

Jemal A, Thomas A, Murray T and Thun M (2002). Cancer statistics, 2002. CA Cancer J. Clin. 52: 23-47.

Lunn RM, Helzlsouer KJ, Parshad R, Umbach DM, et al. (2000). XPD polymorphisms: effects on DNA repair proficiency. Carcinogenesis 21: 551-555.

Maring JG, Wachters FM, Slijfer M, Maurer JM, et al. (2010). Pharmacokinetics of gemcitabine in non-small-cell lung cancer patients: impact of the 79A>C cytidine deaminase polymorphism. Eur. J. Clin. Pharmacol. 66: 611-617.

Park DJ, Stoehlmacher J, Zhang W, Tsao-Wei DD, et al. (2001). A Xeroderma pigmentosum group D gene polymorphism predicts clinical outcome to platinum-based chemotherapy in patients with advanced colorectal cancer. Cancer Res. 61: 8654-8658.

Schiller JH, Harrington D, Belani CP, Langer C, et al. (2002). Comparison of four chemotherapy regimens for advanced non-small-cell lung cancer. N. Engl. J. Med. 346: 92-98.

Seker H, Butkiewicz D, Bowman ED, Rusin M, et al. (2001). Functional significance of XPD polymorphic variants: attenuated apoptosis in human lymphoblastoid cells with the XPD 312 Asp/Asp genotype. Cancer Res. 61: 74307434.

Spitz MR, Wu X, Wang Y, Wang LE, et al. (2001). Modulation of nucleotide excision repair capacity by XPD polymorphisms in lung cancer patients. Cancer Res. 61: 1354-1357.

Takenaka T, Yano T, Kiyohara C, Miura N, et al. (2010). Effects of excision repair cross-complementation group 1 (ERCC1) single nucleotide polymorphisms on the prognosis of non-small cell lung cancer patients. Lung Cancer 67: 101-107.

Tibaldi C, Giovannetti E, Vasile E, Mey V, et al. (2008). Correlation of CDA, ERCC1, and XPD polymorphisms with response and survival in gemcitabine/cisplatin-treated advanced non-small cell lung cancer patients. Clin. Cancer Res. 14: 1797-1803.

Wei SZ, Zhan P, Shi MQ, Shi Y, et al. (2011). Predictive value of ERCC1 and XPD polymorphism in patients with advanced non-small cell lung cancer receiving platinum-based chemotherapy: a systematic review and meta-analysis. Med. Oncol. 28: 315-321.

Wlodarczyk M and Nowicka G (2012). Common polymorphisms in CYP1A1, GSTM1, GSTT1, GSTP1 and XPD genes and endogenous DNA damage. Mol. Biol. Rep. 39: 5699-5704. 